\title{
Single-Nucleotide Polymorphisms Within MicroRNAs Sequences and Their 3' UTR Target Sites May Regulate Gene Expression in Gastrointestinal Tract Cancers
}

\author{
Zahra Saadatian ${ }^{1}$; Andrea Masotti ${ }^{2}$; Ziba Nariman Saleh Fam ${ }^{3}$; Behnam Alipoor ${ }^{4}$; Milad \\ Bastami ${ }^{5,6, *} ;$ Hamid Ghaedi ${ }^{5, "}$ \\ ${ }_{2}^{1}$ Medical Genetics Department, Faculty of Medicine, Tabriz University of Medical Sciences, Tabriz, IR Iran \\ ${ }_{3}^{2}$ Gene Expression-Microarrays Laboratory, IRCCS Bambino Gesu Children's Hospital, Rome, Italy \\ ${ }^{3}$ Medical Genetics Department, Faculty of Medicine, Tehran University of Medical Sciences, Tehran, IR Iran \\ ${ }_{5}^{4}$ Clinical Biochemistry Department, Faculty of Medicine, Tehran University of Medical Sciences, Tehran, IR Iran \\ ${ }^{5}$ Medical Genetics Department, Faculty of Medicine, Shahid Beheshti University of Medical Sciences, Tehran, IR Iran \\ 6 Genomic Research Center, Taleghani Hospital, Shahid Beheshti University of Medical Sciences, Tehran, IR Iran \\ ${ }^{*}$ Corresponding Authors: Milad Bastami, Genomic Research Center, Taleghani Hospital, Shahid Beheshti University of Medical Sciences, Tehran, IR Iran. Tel: +98-2122439959, Fax: \\ +98-2122439961, E-mail: mi.bastami@sbmu.ac.ir; Hamid Ghaedi, Department of Medical Genetics, Faculty of Medicine, Shahid Beheshti University of Medical Sciences, Velenjak st., \\ Shahid Chamran Highway, Tehran, IR Iran. Tel:+98-2122439982, Fax: +98-2122439784, E-mail: h.qaedi@sbmu.ac.ir
}

Received: December 9, 2013; Revised: January 6, 2014; Accepted: March 11, 2014

\begin{abstract}
Background: Esophageal, stomach, and colorectal cancers are commonly lethal gastrointestinal tract (GIT) neoplasms, causing almost two million deaths worldwide each year. some environmental risk factors are acknowledged; however, genetic defects can significantly contribute to predisposition to GIT cancers. Accordingly, recent works have shown that single-nucleotide polymorphisms (SNPs) within miRNAs coding sequence (miR-SNPs) and miRNA target sites (target-SNPs) may further contribute to increased risk of developing cancer. Objectives: In this study, we comprehensively identified miRNA-target gene pairs implicated in GIT cancers and catalogued the presence of potentially functional miR-SNPs and target-SNPs that impair the correct functional recognition.

Materials and Methods: Using bioinformatics tools, manual literature review, and a highly accurate dataset of experimentally validated miRNA-target gene interactions, we compiled a list of miRNA-target genes pairs related to GIT cancers and prioritized them into different groups based on the levels of experimental support. Functional annotations (gene ontology) were applied to these pairs in each group to gain further information.

Results: We identified 97 pairs in which both miRNAs and target genes were implicated in GIT cancers. Several pairs, denoted as highly polymorphic pairs, had both miR-SNPs and target-SNPs. In addition, more than 5000 miRNA-target gene pairs were identified in which, according to the previous reports, either the miRNAs or the target genes had a direct involvement in GIT cancers. More than 800 targetSNPs are located in regulatory regions that were extracted from the ENCODE project through the RegulomeDB database. Of these, 20 were classified as expression quantitative trait loci (eQTLs).

Conclusions: Our work provided a comprehensive source of prioritized and annotated candidate polymorphisms inside miRNAs and their target sites in GIT cancers, which would facilitate the process of choosing right candidate miRNA-target genes and related polymorphisms for future association or functional studies.
\end{abstract}

Keywords:MicroRNAs; Gastrointestinal Neoplasms; Colorectal Neoplasms; Gastric Neoplasms; Esophageal Neoplasms; Single Nucleotide Polymorphisms

\section{Background}

Esophageal, stomach, and colorectal cancers are commonly lethal gastrointestinal tract (GIT) malignancies, causing annually more than 1753000 deaths worldwide (1). Esophageal cancer (EC) is the eighth most common cancer and the sixth leading cause of cancer mortality, with a five-year overall survival rate of $10 \%$ to $16 \%$ (2, 3). Gastric cancer (GC), the fourth most common tumor and the second leading cause of mortality, is often diagnosed in advanced ages and has an average survival rate of only seven to nine months (4-6). Colorectal cancer (CRC), the third most common cancer in men and the

second one in women, is responsible for approximately $8 \%$ of all cancer deaths (7). Despite some well acknowledged environmental risk factors, genetic defects can significantly contribute to predisposition to GIT cancers. MicroRNAs (miRNAs) are a class of small noncoding RNAs that are evolutionary conserved and involved in posttranscriptional regulation of gene expression (8). They modulate gene expression by binding to 3' UTR target sites of mRNAs and repress their translation or promote cleavage and degradation (9). MiRNAs regulate nearly all cellular processes that are altered during tumorigenesis;

Copyright (C) 2014, Iranian Red Crescent Medical Journal; Published by Kowsar Corp. This is an open-access article distributed under the terms of the Creative Commons Attribution License, which permits unrestricted use, distribution, and reproduction in any medium, provided the original work is properly cited. 
their widespread contribution to cancer has been investigated (10). Researchers have obtained an overwhelming amount of data suggesting that single-nucleotide polymorphisms (SNPs) in miRNAs (miR-SNPs) and their target sites (target-SNPs) may be associated with an altered risk of developing cancer (9).

\section{Objectives}

In this work, we comprehensively identified and catalogued miRNA-target gene pairs in GIT cancers and annotated relevant candidate miR-SNPs and their target-SNPs in order to study the potential implications of SNPs in the developing GIT cancers.

\section{Materials and Methods}

We considered esophageal, stomach, and colorectal cancers as representative GIT tumors. Using an in silico approach, we extracted information on SNPs in GIT cancer-related miRNA-target mRNAs. First, we compiled information on the miRNA-target mRNA duplexes with some evidence of contribution into GIT cancers by searching relevant papers in the literature. To this aim, we assembled two lists: one for coding genes and another for miRNAs implicated in GIT cancers; we called them GI-genes (GIT cancers-related genes) and GI-miRNAs lists, respectively. Then, we employed a data set of experimentally validated miRNA-target gene pairs to retain all of the GIT cancer-relevant pairs. Furthermore, we categorized the identified miRNA-target gene pairs into three groups (A, B, and C). Whenever a miRNA-target gene pair was included in GI-miRNAs or GI-genes lists, we assigned the pair to the group-A. Group-B included hosts pairs in which only miRNAs were retrieved from GI-miRNAs list, i.e. target genes were not from GI-gene list. Group-C consisted of pairs in which miRNAs were not from GI-miRNAs list but their target genes belonged to the GI-gene list. Next, we annotated miRNAs-target gene pairs in all of these groups and extracted information about the presence of SNPs in miRNA sequence (miR-SNPs) and in the 3' UTR mRNA sequence (target-SNPs) by using different bioinformatics resources (Table 1). What follows in the remaining of this section is a more detailed description of data sets, resources, and procedures employed in this study.

\begin{tabular}{|c|c|c|}
\hline Tools & URIs & Brief Description \\
\hline miRBase & http://www.mirbase.org/ & $\begin{array}{c}\text { A searchable database of published miRNA sequences and annota- } \\
\text { tion }\end{array}$ \\
\hline TUMIR & $\begin{array}{l}\text { http://www.ncrnalab.com/ } \\
\text { TUMIR/ }\end{array}$ & $\begin{array}{l}\text { An experimentally supported database of miRNA deregulation in } \\
\text { various cancers }\end{array}$ \\
\hline CAMi-Finder & $\begin{array}{c}\text { http://www.isical. } \\
\text { ac.in/ wioinfo_miu/web_isi. } \\
\text { html }\end{array}$ & A Cancer Associated MicroRNA Mining Tool \\
\hline miR2Disease & http://www.mir2disease.org/ & $\begin{array}{l}\text { A comprehensive resource of miRNA deregulation in various hu- } \\
\text { man diseases }\end{array}$ \\
\hline HuGE Navigator & $\begin{array}{l}\text { http://hugenavigator.net/Hu- } \\
\text { GENavigator/home.do }\end{array}$ & $\begin{array}{l}\text { A knowledge base in human genome epidemiology, including } \\
\text { genetic association }\end{array}$ \\
\hline CancerGAMAdb & $\begin{array}{l}\text { http://www.hugenavigator. } \\
\text { net/CancerGEMKB/caIntegra- } \\
\text { torStartPage.do }\end{array}$ & $\begin{array}{l}\text { A continually updated database of genetic association studies in } \\
\text { cancer }\end{array}$ \\
\hline Cancer Gene Census & $\begin{array}{l}\text { http://cancer.sanger.ac.uk/can- } \\
\text { cergenome/projects/census/ }\end{array}$ & $\begin{array}{c}\text { A catalog of those genes for which mutations have been causally } \\
\text { implicated in cancer }\end{array}$ \\
\hline $\begin{array}{l}\text { Network of Cancer Genes } \\
\text { (NCG4) }\end{array}$ & http://bio.ieo.eu/ncg/ & Reports annotation on 2000 protein-coding cancer genes \\
\hline DAVID & http://david.abcc.ncifcrf.gov/ & A comprehensive set of functional annotation tools \\
\hline Gene Expression Atlas & http://www.ebi.ac.uk/gxa/ & $\begin{array}{c}\text { Provides information on gene expression patterns under different } \\
\text { biological conditions }\end{array}$ \\
\hline dbGaP & $\begin{array}{l}\text { http://www.ncbi.nlm.nih.gov/ } \\
\text { sites/entrez?db=gap }\end{array}$ & $\begin{array}{c}\text { An archive of the results of studies investigating interaction of } \\
\text { genotypes and phenotypes }\end{array}$ \\
\hline NHGRI GWAS catalog & www.genome.gov/gwastudies & A Catalog of Published Genome-Wide Association Studies \\
\hline RegulomeDB & http://www.regulomedb.org/ & $\begin{array}{l}\text { A database that annotates SNPs with known and predicted regula- } \\
\text { tory elements }\end{array}$ \\
\hline PolymiRTS 3.0 & http:/|www.regulomedb.org/ & A database of SNPs in microRNAs and their target sites \\
\hline
\end{tabular}

\footnotetext{
a Abbreviations: SNP, single-nucleotide polymorphisms; and mi RNA, microRNA.
} 
Saadatian Z et al.

\subsection{Gastrointestinal Tract Cancers-Related Genes}

This list contained GI-genes extracted from the Cancer Gene Census (11), candidate genes extracted from the Cancer Gene Network (version 4) (12), which is a collection of manually curated genes from 77 whole genome or whole exome cancer resequencing experiments, and candidate genes from Cancer Genome-wide Association and Metaanalysis Database (CancerGAMAdb), which is a database of Genome-wide Association Studies (GWAS) and metaanalysis data in cancer (13).

\subsection{Gastrointestinal Tract Cancers-Related Mi- CroRNAS}

In the GI-miRNAs list, we included miRNA with at least one of the following essential characteristics:

- Altered expression in GIT cancers.

- Evidence for association of a miRNA-hosted-SNP with GIT cancers or their outcome.

Data for altered expression of miRNAs were retrieved from TUMIR (14), CAMi-Finder, miR2 disease (15), and from the literature $(2,4,7,16,17)$. For associations, we used HuGE Navigator (last updated on November 29, 2013), an integrated database of genetic associations (13). MiRNA coordinates were retrieved from miRBase (release 20) $(18,19)$.

\subsection{MicroRNA-Target Gene Interactions}

Recently, a technique, named CLASH (crosslinking, ligation, and sequencing of hybrids), has been developed for ligation and sequencing of miRNA-target RNA duplexes associated with human AGO1 (20). Using CLASH, Helwak et al. have reported a data set of more than 18000 highconfidence miRNA-mRNA interactions, which represents a breakthrough in the field (20). Therefore, we took advantage of CLASH datasets as a source of experimentally validated miRNA-target interactions. The coordinates of SNPs in binding sites where retrieved from PolymiRTS 3.0 database (21).

\subsection{Functional Annotation Analysis}

Gene list functional analysis was performed by the Database for Annotation, Visualization and Integrated Discovery (DAVID v. 6.7) with the EASE score threshold, i.e. P Value, being 0.05 and count threshold, i.e. minimum number of genes for corresponding term being set at two $(22,23)$.

\subsection{Differential Gene Expression Data Sets}

Gene Expression Atlas was searched for upregulated or downregulated genes in GIT tumors by searching terms such as colon cancer, colorectal cancer, gastric cancer, and esophageal cancers (24). The differential expression of genes reported in four previous studies (experiment accession IDs: E-GEOD-13471, E-GEOD-1420, E-GEOD-19249, E-GEOD-19826, E-GEOD-23878, E-GEOD-2685, E-MTAB-57,
E-MTAB-62, and E-MTAB-145) were downloaded and employed in this work (25-33).

\subsection{The Encyclopedia of DNA Elements and Expres- sion Quantitative Trait Loci Data}

RegulomeDB is a database that annotates SNPs with known and predicted regulatory elements in the intergenic regions of the Homo sapiens genome. These elements include regions of DNAase hypersensitivity, binding sites of transcription factors, and promoter regions. The source of these data includes public datasets from GEO, the Encyclopedia of DNA Elements (ENCODE) project, and published literature (34). We employed this database to find target-SNPs located in annotated regions and test their function as expression quantitative trait loci (eQTLs).

\section{Results}

\subsection{Group-A (GI-miRNA:GI-Gene Pairs)}

We identified a total of 36 GI-miRNAs, altogether regulating 66 GI-genes in the form of 97 unique miRNA-target duplexes. Searching for SNPs inside miRNAs sequences and mRNA-binding sites in this group, we identified 29 miR-SNPs (25 with frequency information) and 150 target-SNPs (61 with frequency information). Among these, we looked for "highly polymorphic pairs", which bore both miR-SNP and target-SNP. We found several notable examples, including hsa-miR-93-5p:BIRC5 and hsa-miR-149$5 p$ :BIRC5. Survivin gene (BIRC5) belongs to inhibitor of apoptosis gene family and is implicated in GIT cancers. On the other hand, both regulators of survivin ( $h s a-m i R-$ 93-5p and hsa-miR-149-5p) are also involved in GC. In the highly polymorphic pairs we identified hsa-miR-196a-5p and $h$ sa-miR-92-3p. The first one seems to be involved in all GIT cancer types, and the second has a crucial role in CRC and GC, by regulating 20 GI-genes, 14 of which had SNPs in binding sites (Table 2). In addition, hsa-miR-222-3p and hsa-miR-100-5p were involved in two GIT cancers (CRC/GC and CRC/EC respectively).

\subsection{Group-B (GI-miRNA:Non-GI-Genes)}

This group comprises 7763 interactions including 83 unique GI-miRNAs and 4549 unique non-GI-target genes. We tested the hypothesis that some target genes in group-B might have functional relevance to GIT cancers. By performing functional annotation analysis to the GI-genes list and group-B target genes, we found that about $64 \%$ of targets in group-B were involved in the same biological processes as GI-genes. These genes were kept in the list of potential candidate genes. The five most enriched processes in GI-genes list were positive regulation of cell differentiation (GO: 0045597), enzyme linked receptor protein signaling pathway (GO: 0007167), regulation of programmed cell death (GO: 0043067), phos- 
phate metabolic process (GO: 0006796), and cell surface receptor-linked signal transduction (GO: 0007166). To further support these evidences, we explored differential gene expression data sets of Gene Expression Atlas to identify upregulated or downregulated genes in GIT tumor in comparison with normal tissues. Intersection analysis of differentially expressed genes and potential candidate genes indicated that more than $94 \%$ (2745 of 2904) of them were differentially expressed in at least one GIT tumor.

\begin{tabular}{|c|c|c|c|c|c|}
\hline MiRNA & $\begin{array}{l}\text { MiRNA Alteration } \\
\text { in GIT Cancers }\end{array}$ & MiR-SNP & $\begin{array}{l}\text { Number of Targets } \\
\text { With Target-SNPs }\end{array}$ & $\begin{array}{c}\text { Number of } \\
\text { Target-SNPs }\end{array}$ & GIT Cancer \\
\hline hsa-miR-148b-3p & CRC (down) & rs74878365 & 2 & 2 & CRC \\
\hline hsa-miR-149-5p & GC (association) & rs2292832; rs71428439 & 2 & 3 & GC, EC, and CRC \\
\hline hsa-miR-15b-5p & GC (up) & rs146020563; rs192595529 & 1 & 1 & GC \\
\hline hsa-miR-16-5p & CRC (up) & rs72631826 & 3 & 4 & CRC \\
\hline hsa-miR-182-5p & CRC (up) & rs76481776; rs77586312; rs80041074 & 1 & 1 & CRC \\
\hline hsa-miR-194-5p & CRC (down) & rs11231898 & 1 & 2 & CRC \\
\hline hsa-miR-196a-5p & $\begin{array}{l}\text { GC and EC (up); CRC, } \\
\text { GC, and EC (associa- } \\
\text { tion) }\end{array}$ & $\begin{array}{l}\text { rs11614913; rs185070757; rs186449583; } \\
\text { rs190478598; }\end{array}$ & 2 & 4 & GC, EC, and CRC \\
\hline hsa-miR-20a-5p & CRC (up) & rs185831554 & 1 & 2 & CRC \\
\hline hsa-miR-222-3p & CRC and GC (up) & rs187612279; rs191727254; rs72631825 & 3 & 9 & CRC and GC \\
\hline hsa-miR-26a-5p & CRC (association) & rs182070256; rs190898565 & 3 & 4 & CRC and GC \\
\hline hsa-miR-30a-3p & CRC (down) & rs149150037; rs190842689 & 1 & 2 & CRC \\
\hline hsa-miR-30b-5p & GC (up) & rs111424617 & 1 & 2 & GC \\
\hline hsa-miR-605-5p & CRC (association) & rs113212828; rs2043556 & 1 & 3 & CRC \\
\hline hsa-miR-92a-3p & $\begin{array}{c}\text { CRC (up/down) and } \\
\text { GC (up) }\end{array}$ & rs9589207 & 14 & 42 & CRC and GC \\
\hline hsa-miR-93-5p & GC (up) & rs72631824 & 1 & 2 & GC, EC, and CRC \\
\hline
\end{tabular}

a Abbreviations: CRC, colorectal cancer; Down, downregulated; EC, esophageal cancer; GC, gastric cancer; and Up, upregulated.

$\mathrm{b}$ The table shows miRNA of each highly polymorphic pair, its alteration in cancer and relevant pre-miRNA SNPs, the number of its target with SNPs in their binding sites, and the total number of SNPs in these binding sites. The last column shows involvement of target genes bearing target-SNPs in GIT cancers.

Table 3. Clinically Important Target-SNPs With Evidence of Disease Association ${ }^{\text {a }}$

\begin{tabular}{|c|c|c|c|c|c|}
\hline Target & Target-SNP & Disease & MiRNA & $\begin{array}{l}\text { MiRNA Alteration } \\
\text { in GIT Cancers; } \\
\text { "Expression or Asso- } \\
\text { ciation" } \\
\end{array}$ & Reference \\
\hline BID & rs8190315 & Breast cancer & hsa-miR-92a-3p & CRC (down), GC (up) & (35) \\
\hline ERCC1 & rs3212986 & Multiple cancers & hsa-miR-92a-3p & CRC (down), GC (up) & (36) \\
\hline ITPA & rs1127354 & $\begin{array}{l}\text { Ribavirin-induced anemia and out- } \\
\text { comes of therapy in patients infected } \\
\text { with HCV }\end{array}$ & hsa-miR-93-5p & GC (up) & $(37,38)$ \\
\hline ZIC2 & rs13542 & Orofacial clefts & hsa-miR-320a & CRC (up), GC (up) & (39) \\
\hline BET1L & rs2280543 & Intracranial aneurysm, uterine fibroids & hsa-miR-320a & CRC (up), GC (up) & $(40,41)$ \\
\hline MAPRE1 & rs7270085 & $\begin{array}{l}\text { Attention deficit hyperactivity disorder } \\
\text { (combined symptoms) }\end{array}$ & hsa-miR-92a-3p & $\begin{array}{l}\text { CRC (up/down), GC } \\
\text { (up) }\end{array}$ & $(42)$ \\
\hline HDAC7 & rs75365750 & Obesity-related traits & hsa-miR-149-5p & GC (association) & $(43)$ \\
\hline ESPL1 & rs1318648 & Chronic lymphocytic leukemia & hsa-miR-149-5p & GC (association) & $(44)$ \\
\hline WWP2 & rs3748386 & Myocardial infarction & hsa-let-7a-5p & CRC (up) & $\begin{array}{c}\text { Genetic Association } \\
\text { Database }\end{array}$ \\
\hline DLD & rs4518 & Cardiovascular diseases, P-selectin & hsa-miR-99a-5p & $\mathrm{EC}$ (down) & $(45)$ \\
\hline
\end{tabular}

a Abbreviations: CRC, colorectal cancer; Down: downregulated; EC, esophageal cancer; GC, gastric cancer; and Up, upregulated. 
Saadatian Z et al.

\begin{tabular}{|c|c|c|c|c|c|c|c|c|}
\hline Target Gene & Target-SNP & miRNA & Group & eQTL & $\mathrm{TF}^{\mathrm{a}}$ Binding & TF Motif & DNase Footprint & DNase Peak \\
\hline HIST1H2AL & rs200981 & hsa-miR-10b-5p & B & + & + & + & + & + \\
\hline HIST1H2AL & rs41448545 & hsa-miR-10b-5p & B & + & + & + & + & + \\
\hline ARCN1 & rs17742 & hsa-miR-92a-3p & B & + & + & + & + & + \\
\hline RPS16 & rs17626 & hsa-miR-17-3p & B & + & + & + & + & + \\
\hline H1Fo & rs6000898 & hsa-let-7a-5p & B & + & + & + & - & + \\
\hline MCM7 & rs2307355 & hsa-miR-503-5p & B & + & + & - & - & + \\
\hline RPL27A & rs6735 & hsa-miR-342-3p & B & + & + & - & - & + \\
\hline GAPDH & rs1803621 & hsa-miR-149-5p & B & + & + & - & - & + \\
\hline TUBA1B & rs2753 & hsa-miR-320a & B & + & + & - & - & + \\
\hline GCN1L1 & rs2286050 & hsa-miR-423-3p & B & + & + & - & - & + \\
\hline KIF23 & rs11852675 & $h s a-m i R-25-3 p$ & B & + & + & - & - & + \\
\hline MYO1D & rs2285428 & hsa-miR-320a & B & + & + & - & - & + \\
\hline ACTG1 & rs11549223 & hsa-miR-221-3p & B & + & + & - & - & + \\
\hline NR2F6 & rs2288539 & hsa-miR-196a-5p & B & + & + & - & - & + \\
\hline XPO4 & rs4617691 & hsa-miR-18a-3p & B & + & + & - & - & - \\
\hline PEX6 & rs1129187 & hsa-miR-149-5p & B & + & - & - & - & + \\
\hline SOD2 & rs7855 & hsa-miR-769-3p & $\mathrm{C}$ & + & - & - & - & + \\
\hline PDHX & rs497582 & hsa-miR-181b-5p & B & + & - & - & - & + \\
\hline WWP2 & rs3748386 & hsa-let-7a-5p & B & + & - & - & - & + \\
\hline MYO19 & rs2306590 & hsa-miR-615-3p & $\mathrm{C}$ & + & - & - & - & + \\
\hline
\end{tabular}

Consequently, the primary list of 7763 pairs were reduced to 4856 pairs (78 miRNAs and 2745 target genes), which totally hosted 55 miR-SNPs ( 45 with frequency information) and 6562 target-SNPs (2857 with frequency information).

To gain further insights into the potential functional relevance of target-SNPs, we searched the dbGaP (46), $\mathrm{NH}$ GRI GWAS catalog (47), and Genetic Association Database (GAD) (48) to extract information on their association with diseases. Interestingly, association of eleven SNPs with diseases were previously reported (Table 3) (37-45). Among these SNPs, rs8190315 and rs3212986 are located in $B I D$ and ERCC1, respectively. These two regions are recognized by hsa-miR-92a-3p, a miRNA that is downregulated in CRC and upregulated in GC.

\subsection{Group-C (Non-GI-miRNA:GI-Gene Pairs)}

MiRNAs that regulate GI-genes were assigned to this group, which contains 214 unique pairs (68 miRNAs and 107 target genes). We identified 45 miR-SNPs (37 with frequency information) and 294 target-SNPs (133 with frequency information).

\subsection{Annotation of Target-SNPS}

Using RegulomeDB, we annotated target-SNPs in groups A, B, and C. A total of 815 target-SNPs overlap with transcription factor binding sites and DNase I hypersensitive regions. Out of these 815 target-SNPs, 24 reside in transcription factor motifs and/or footprints. Furthermore, we found 20 target-SNPs that function as eQTLs (Table 4).

\section{Discussion}

Increasing evidences propose that SNPs within miRNAs and their 3' UTR binding sites may play active roles in a variety of human diseases, especially GIT cancers. Here, we catalogued miRNA-target gene pairs with varying levels of implications for esophageal, gastric and colorectal tumors, and annotated the presence of potentially functional SNPs. We obtained a list of more than 5100 GIT cancers-related miRNA-target gene pairs, hosting 91 miR-SNPs and 7006 target-SNPs, and prioritized them according to experimental findings.

In group-A, we demonstrated several novel GIT cancers-related interactions and made a list of highly polymorphic pairs. MiR-SNP can alter the expression level of the wild-type miRNA, whereas the presence of a functional SNP in the $3^{\prime}$ UTR target site may potentially affect the binding with a specific miRNAs and alter the posttranscriptional regulation by miRNAs. Therefore, we 
concluded that it would be a good practice to examine the effects generated by the presence of miR-SNPs and target-SNPs in highly polymorphic pairs when studying their contribution to the cancers development or evaluating the predisposition to it. Overall, 97 miRNAtarget pairs and their 86 SNPs identified in this group represent prioritized candidate pairs to be considered in further experimental studies for their high probability of involvement in an impaired modulation of gene expression.

Regarding pairs in group-B, we showed that a considerable proportion of target genes are involved in the biological processes altered in GIT cancers and are differentially expressed in these tumors. Incidentally, we further explored the functional roles of some of these variants by indicating that 11 SNPs with strong evidence of association with a variety of diseases ranging from breast cancer to orofacial cleft actually are located inside binding sites of clinically important miRNAs. The most noteworthy SNP may be rs3212986 in ERCC1 gene with reported association with multiple cancer types including, but not limited to, estrogen-related cancers (breast, cervical, and ovarian), smoking-related cancers (lung, esophageal, bladder, head and neck, and pancreatic cancer), and brain tumors. Therefore, a large number of pairs and polymorphisms found in group-B, which were not explored in this work, could represent a further line of research in a near future.

Regarding group-C, our results showed that several miRNAs regulating GI-genes could be potentially relevant and could offer the opportunity to detect other novel candidate miRNAs. Since variations in genomic regulatory regions have crucial functional effects, we exploited the information of the ENCODE project to classify the target-SNPs obtained in our work. Table 4 presents twenty target-SNPs in the context of ENCODE.

In summary, our data provided a comprehensive source of prioritized and annotated candidate polymorphisms within miRNAs sequences and their 3' UTR target sites in GIT cancers, which could facilitate the process of selecting the right candidate miRNA-target genes for functional studies and focusing on potentially relevant polymorphisms for further association studies.

\section{Authors' Contributions}

Study concept and design: Milad Bastami, Hamid Ghaedi, and Andrea Masotti. Data collection and workflow preparation: Zahra Saadatian, Ziba Nariman-Saleh-Fam, and Andrea Masotti. Analysis of data: Zahra Saadatian, Ziba Nariman-Saleh-Fam, and Milad Bastami. Manuscript drafting: Ziba Nariman-Saleh-Fam and Zahra Saadatian. Manuscript editing: Andrea Masotti, Milad Bastami, and Hamid Ghaedi.

\section{References}

1. Jemal A, Bray F, Center MM, Ferlay J, Ward E, Forman D. Global cancer statistics. CA Cancer J Clin. 2011;61(2):69-90.

2. Mayne GC, Hussey DJ, Watson DI. MicroRNAs and esophageal cancer--implications for pathogenesis and therapy. Curr Pharm Des. 2013;19(7):1211-26.

3. Akbari MR, Malekzadeh R, Shakeri R, Nasrollahzadeh D, Foumani M, Sun Y, et al. Candidate gene association study of esophageal squamous cell carcinoma in a high-risk region in Iran. Cancer Res. 2009;69(20):7994-8000.

4. Pan HW, Li SC, Tsai KW. MicroRNA dysregulation in gastric cancer. Curr Pharm Des. 2013;19(7):1273-84.

5. Najafi F, Mozaffari HR, Karami M, Izadi B, Tavvafzadeh R, Pasdar Y Trends in incidence of gastrointestinal tract cancers in Western iran, 1993-2007. Iran Red Crescent Med J. 2011;13(11):805-10.

6. Anbiaee R, Mojir Sheibani K, Torbati P, Jaam H. Abnormal expression of e-cadherin in gastric adenocarcinoma, and its correlation with tumor histopathology and helicobacter pylori infection. Iran Red Crescent Med J. 2013;15(3):218-22.

7. Dassow H, Aigner A. MicroRNAs (miRNAs) in colorectal cancer: from aberrant expression towards therapy. Curr Pharm Des. 2013;19(7):1242-52.

8. Bartel DP. MicroRNAs: genomics, biogenesis, mechanism, and function. Cell. 2004;116(2):281-97.

9. Ryan BM, Robles AI, Harris CC. Genetic variation in microRNA networks: the implications for cancer research. Nat Rev Cancer. 2010;10(6):389-402.

10. Olive V, Jiang I, He L. mir-17-92, a cluster of miRNAs in the midst of the cancer network. Int J Biochem Cell Biol. 2010;42(8):1348-54.

11. Futreal PA, Coin L, Marshall M, Down T, Hubbard T, Wooster $\mathrm{R}$, et al. A census of human cancer genes. Nat Rev Cancer. 2004;4(3):177-83.

12. Syed AS, D'Antonio M, Ciccarelli FD. Network of Cancer Genes: a web resource to analyze duplicability, orthology and network properties of cancer genes. Nucleic Acids Res. 2010;38(Database issue):D670-5.

13. Yu W, Gwinn M, Clyne M, Yesupriya A, Khoury MJ. A navigator for human genome epidemiology. Nat Genet. 2008;40(2):124-5.

14. Dong L, Luo M, Wang F, Zhang J, Li T, Yu J. TUMIR: an experimentally supported database of microRNA deregulation in various cancers. JClin Bioinforma. 2013;3(1):7.

15. Jiang Q, Wang Y, Hao Y, Juan L, Teng M, Zhang X, et al. miR2Disease: a manually curated database for microRNA deregulation in human disease. Nucleic Acids Res. 2009;37(Database issue):D98-104.

16. Shen J, Wu WK, Ren SX, Zhang L, Chan RL, Wong CC, et al. miRNAs in gastrointestinal and liver cancers: their perspectives and clinical applications. Curr Pharm Des. 2013;19(7):1301-10.

17. Song S, Ajani JA. The role of microRNAs in cancers of the upper gastrointestinal tract. Nat Rev Gastroenterol Hepatol. 2013;10(2):109-18.

18. Griffiths-Jones S. The microRNA Registry. Nucleic Acids Res 2004;32(Database issue):D109-11.

19. Kozomara A, Griffiths-Jones S. miRBase: integrating microRNA annotation and deep-sequencing data. Nucleic Acids Res. 2011;39(Database issue):D152-7.

20. Helwak A, Kudla G, Dudnakova T, Tollervey D. Mapping the human miRNA interactome by CLASH reveals frequent noncanonical binding. Cell. 2013;153(3):654-65.

21. Bhattacharya A, Ziebarth JD, Cui Y. PolymiRTS Database 3.0: linking polymorphisms in microRNAs and their target sites with human diseases and biological pathways. Nucleic Acids Res. 2014;42(Database issue):D86-91.

22. Huang da W, Sherman BT, Lempicki RA. Bioinformatics enrichment tools: paths toward the comprehensive functional analysis of large gene lists. Nucleic Acids Res. 2009;37(1):1-13.

23. Huang da W, Sherman BT, Lempicki RA. Systematic and integrative analysis of large gene lists using DAVID bioinformatics resources. Nat Protoc. 2009;4(1):44-57.

24. Kapushesky M, Emam I, Holloway E, Kurnosov P, Zorin A, Malone J, et al. Gene expression atlas at the European bioinformatics institute. Nucleic Acids Res. 2010;38(Database issue):D690-8.

25. Abdueva D, Wing M, Schaub B, Triche T, Davicioni E. Quantitative expression profiling in formalin-fixed paraffin-embedded samples by affymetrix microarrays. J Mol Diagn. 2010;12(4):409-17.

26. Ancona N, Maglietta R, Piepoli A, D'Addabbo A, Cotugno R, Savino 
$\mathrm{M}$, et al. On the statistical assessment of classifiers using DNA microarray data. BMC Bioinformatics. 2006;7:387.

27. Hippo Y, Taniguchi H, Tsutsumi S, Machida N, Chong JM, Fukayama M, et al. Global gene expression analysis of gastric cancer by oligonucleotide microarrays. Cancer Res. 2002;62(1):233-40.

28. Irizarry RA, Ladd-Acosta C, Wen B, Wu Z, Montano C, Onyango P, et al. The human colon cancer methylome shows similar hypoand hypermethylation at conserved tissue-specific CpG island shores. Nat Genet. 2009;41(2):178-86.

29. Kimchi ET, Posner MC, Park JO, Darga TE, Kocherginsky M, Karrison T, et al. Progression of Barrett's metaplasia to adenocarcinoma is associated with the suppression of the transcriptional programs of epidermal differentiation. Cancer Res. 2005;65(8):3146-54.

30. Lukk M, Kapushesky M, Nikkila J, Parkinson H, Goncalves A, Huber W, et al. A global map of human gene expression. Nat Biotechnol. 2010;28(4):322-4.

31. Uddin S, Ahmed M, Hussain A, Abubaker J, Al-Sanea N, AbdulJabbar A, et al. Genome-wide expression analysis of Middle Eastern colorectal cancer reveals FOXM1 as a novel target for cancer therapy. Am J Pathol. 2011;178(2):537-47.

32. Vaquerizas JM, Kummerfeld SK, Teichmann SA, Luscombe NM. A census of human transcription factors: function, expression and evolution. Nat Rev Genet. 2009;10(4):252-63.

33. Wang Q, Wen YG, Li DP, Xia J, Zhou CZ, Yan DW, et al. Upregulated INHBA expression is associated with poor survival in gastric cancer. Med Oncol. 2012;29(1):77-83.

34. Boyle AP, Hong EL, Hariharan M, Cheng Y, Schaub MA, Kasowski $M$, et al. Annotation of functional variation in personal genomes using RegulomeDB. Genome Res. 2012;22(9):1790-7.

35. Gaudet MM, Milne RL, Cox A, Camp NJ, Goode EL, Humphreys MK, et al. Five polymorphisms and breast cancer risk: results from the Breast Cancer Association Consortium. Cancer Epidemiol Biomarkers Prev. 2009;18(5):1610-6.

36. Zhang L, Wang J, Xu L, Zhou J, Guan X, Jiang F, et al. Nucleotide excision repair gene ERCC1 polymorphisms contribute to cancer susceptibility: a meta-analysis. Mutagenesis. 2012;27(1):67-76.

37. Fellay J, Thompson AJ, Ge D, Gumbs CE, Urban TJ, Shianna KV, et al. ITPA gene variants protect against anaemia in patients treated for chronic hepatitis C. Nature. 2010;464(7287):405-8.

38. Ochi H, Maekawa T, Abe H, Hayashida Y, Nakano R, Kubo M, et al.
ITPA polymorphism affects ribavirin-induced anemia and outcomes of therapy--a genome-wide study of Japanese HCV virus patients. Gastroenterology. 2010;139(4):1190-7.

39. Ludwig KU, Mangold E, Herms S, Nowak S, Reutter H, Paul A, et al. Genome-wide meta-analyses of nonsyndromic cleft lip with or without cleft palate identify six new risk loci. Nat Genet. 2012;44(9):968-71.

40. Cha PC, Takahashi A, Hosono N, Low SK, Kamatani N, Kubo M et al. A genome-wide association study identifies three loci associated with susceptibility to uterine fibroids. Nat Genet. 2011;43(5):447-50.

41. Low SK, Takahashi A, Cha PC, Zembutsu H, Kamatani N, Kubo M, et al. Genome-wide association study for intracranial aneurysm in the Japanese population identifies three candidate susceptible loci and a functional genetic variant at EDNRA. Hum Mol Genet. 2012;21(9):2102-10.

42. Ebejer JL, Duffy DL, van der Werf J, Wright MJ, Montgomery G, Gillespie NA, et al. Genome-wide association study of inattention and hyperactivity-impulsivity measured as quantitative traits. Twin Res Hum Genet. 2013;16(2):560-74.

43. Comuzzie AG, Cole SA, Laston SL, Voruganti VS, Haack K, Gibbs $\mathrm{RA}$, et al. Novel genetic loci identified for the pathophysiology of childhood obesity in the Hispanic population. PLoS One. 2012;7(12).

44. Rasi S, Spina V, Bruscaggin A, Vaisitti T, Tripodo C, Forconi F, et al A variant of the LRP4 gene affects the risk of chronic lymphocytic leukaemia transformation to Richter syndrome. Br J Haematol. 2011;152(3):284-94.

45. Benjamin EJ, Dupuis J, Larson MG, Lunetta KL, Booth SL, Govindaraju DR, et al. Genome-wide association with select biomarker traits in the Framingham Heart Study. BMC Med Genet. 2007;8 Suppl 1:S11.

46. Mailman MD, Feolo M, Jin Y, Kimura M, Tryka K, Bagoutdinov R, et al. The NCBI dbGaP database of genotypes and phenotypes. Nat Genet. 2007;39(10):1181-6.

47. Hindorff L, MacArthur J, Morales J, Junkins H, Hall P, Klemm A. Division of Genomic Medicine A Catalog of Published Genome-Wide Association Studies. Available from: http:/|www.genome.gov/ gwastudies/.

48. Becker KG, Barnes KC, Bright TJ, Wang SA. The genetic association database. Nat Genet. 2004;36(5):431-2. 